\title{
Heavy quarkonium dissociation at finite temperature with effective field theories
}

\section{Miguel Angel Escobedo*}

Physik-Department, Technische Universität München, James-Franck-Str. 1, 85748, Germany

E-mail: miguel.escobedo@ph.tum.de

\begin{abstract}
The dissociation of heavy quarkonium seen in heavy-ion collisions is a phenomena that allows to extract information of the produced thermal medium. This was believed to be due to the screening of the static potential, but recently perturbative computations and some lattice studies have pointed out to the possibility of having an imaginary part of the potential that would also contribute to dissociation. In recent years a program to study heavy quarkonium with the use of non-relativistic effective field theories has been started, this allows to make the computations in a more systematic way by defining a more suitable power counting and making it more difficult to miss necessary resummations. With these techniques the picture of the imaginary potential has been confirmed and a complete study from very low temperatures up to dissociation has been performed for the perturbative case, finding the different energy shifts and decay widths for the different temperature regimes. In these proceedings a review of these results will be given, with special focus on the more recent developments, as the connection between the imaginary part and the cross-section used in phenomenological models and the effect of having a finite velocity between the medium and the heavy quarkonium.
\end{abstract}

Xth Quark Confinement and the Hadron Spectrum

8-12 October 2012

TUM Campus Garching, Munich, Germany

\footnotetext{
* Speaker.
} 


\section{Introduction}

Since the original proposal of [1] a lot of effort has been devoted to the study of heavy quarkonium as a signal of deconfinement. Recently several groups have started to use the ideas of Effective Field Theories (EFT), which has been very successful in the vacuum (see [2] and references therein), to the problem of finite temperature Quarkonia [3,4]. This approach exploits the fact that there are three different and well separated energy scales in heavy quarkonium because it is a non-relativistic system. These scales are the mass of the heavy quark $m$, the inverse of the typical radius $1 / r$ and the binding energy $E$, and they fulfil $m \gg 1 / r \gg E$. To highlight the convenience of using these techniques let us consider the example of the computation of the binding energy $E$, if we compute this quantity directly using $\mathrm{pQCD}$ we would obtain a perturbative series

$$
E=m \alpha_{s} \sum_{n=0}^{\infty} \alpha_{s}^{n} A_{n}(v)
$$

where $A_{n}$ are the coefficients of the perturbative expansion that depend on the velocity around the center of mass $v$. In a non-relativistic system as heavy quarkonium $v$ is small and nothing prevents $A_{n}$ to go to $\infty$ as $v \rightarrow 0$. When this happens we lose control of the naive perturbative expansion. This problems is automatically solved when using EFT because one can define a power counting in such a way that the computation is organized in powers of both $\alpha_{s}$ and $v$.

When the temperature is introduced, one has to take into account how big or small are the temperature induced energy scales compared to the ones that already appear at $T=0$. For $T \gg$ $1 / r \sim m_{D}$, where $m_{D}$ the Debye mass, the computations in EFT [3,4] confirmed the existence of an imaginary part in the perturbative static potential first computed in [5], identified as the mean dissociation mechanism. In the $1 / r \gg T$ case the EFT computations $[3,4,6]$ found next-to-leading order corrections both to the binding energy and to the decay width which can be important for LHC physics and in the particular case of $1 / r \gg T \gg E \gg m_{D}$ they have been compared with lattice computations finding a reasonable agreement [7].

On [8] a review of the application of EFTs to heavy quarkonium at finite temperature can be found. Here we focus on more recent applications of these techniques. First we are going to study in section 2 the relation of the decay widths and imaginary parts of potentials that can be found using EFTs with the cross-section that are often used in phenomenological studies. After this we are going to discuss in section 3 how thermal effects are modified when heavy quarkonium is moving with a finite momentum with respect to the thermal bath. Finally the conclusions will be given in section 4 .

\section{From decay widths to cross sections.}

In the EFT approach two different mechanism that dissociate heavy quarkonium were identified [4], the singlet-to-octet thermal break-up and the effect of Landau damping on the static potential. However, previous studies in the literature used a different method to compute this decay width. First, a perturbative cross section $\sigma$ is used. The perturbative cross section may be modified, for example, to use thermal masses instead of the vacuum values or to take $\alpha_{s}$ as a free parameter. Then this cross section is related with the decay width $\Gamma$ by convoluting it with the corresponding 
parton distribution function, $n(k)$

$$
\Gamma=\int \frac{d^{3} k}{(2 \pi)^{3}} n(k) \sigma(k) .
$$

Using this approach two different mechanism were identified, the gluo-dissociation [9] with corresponds to the process $g+H Q \rightarrow Q+\bar{Q}$ (HQ=Heavy quarkonium), and the quasi-free dissociation [10] which corresponds to the process $p+H Q \rightarrow p+Q+\bar{Q}$ where $p$ is a parton that can be either a light quark or a gluon. These two mechanisms have been used in a number of phenomenological comparisons to the LHC data, see Ref. [11, 12, 13, 14].

The aim of this section is to determine the relation between these two different approaches and find the cross section that can reproduce the EFT results. This will clarify which additional information is obtained by using EFT and also the relation between the quasi-free dissociation cross section and the imaginary part of the potential.

\subsection{Gluo-dissociation}

Gluo-dissociation $(g+H Q \rightarrow Q+\bar{Q})$ was identified as a relevant mechanism for the inmedium decay of Quarkonia in [9]. There they use for the cross section the computation found in [15]. Several approximations were done in doing this. First, the interaction between the heavy quarkonium and the gluon is taken as a color dipole. Indeed, in the EFT approach one uses in this situation pNRQCD [16] in which a multipole expansion of the gauge fields is performed, which at this level of accuracy is equivalent to a color dipole approximation. A second approximation that was done in [15] was to use the large $N_{c}$ limit, in this case what this assumption does is to suppress the interaction between the $Q$ and $\bar{Q}$ that appear in the final state because in the large $N_{c}$ limit the octet potential vanish.

In the EFT computation a decay width in the $T \sim E$ regime [3] was found, which can be identified with the effect of gluo-dissociation

$$
\Gamma_{n}=\frac{g^{2} C_{F}}{3 \pi}\left\langle n\left|r^{i}\right| E_{1}-\left.h_{o}^{(0)}\right|^{3} n_{B}\left(\left|E_{1}-h_{o}^{(0)}\right|\right) r^{i} \mid n\right\rangle,
$$

where $n$ is a compact notation to denote all the relevant quantum numbers of heavy quarkonium and $h_{0}^{(0)}$ is the octet Hamiltonian [16]. The expectation value can be analytically evaluated in the $T \gg E$ limit, see [4]. To obtain a cross section from this formula one can make use of the cutting rules, that also have a finite temperature version [17]. Although the situation is more complicated when higher orders in perturbation theory are considered, for the diagram we are considering the application of these cutting rules are quite analogous to what one founds at zero temperature. The more relevant change is that when integrating over the phase space of the decay one has to multiply by the $n_{B}$ Bose-Einstein ( $n_{F}$ Fermi-Dirac) distribution function for in-coming gluons (fermions) and by $1+n_{B}\left(1-n_{F}\right)$ for out-going gluons (fermions). For gluo-dissociation the expected structure is indeed the one shown in eq. (2.1). This can be seen by an explicit computation in pNRQCD, by computing the imaginary part of the singlet self-energy one obtains a width's structure of the following type

$$
\Gamma_{n}=\int \frac{d^{3} k}{(2 \pi)^{3}} n_{B}(k)\langle n|\Sigma(k)| n\rangle .
$$


If the integration over $k$ is performed, the result in eq. (2.2) is recovered. However, one can also identify the cross sections, such as for the $1 \mathrm{~S}$ state,

$$
\sigma_{1 S}=\langle 1 S|\Sigma(k)| 1 S\rangle
$$

which can be computed explicitly by using color octet wave functions, yielding

$$
\sigma_{1 S}^{g d}(k)=\frac{\alpha_{s} C_{f}}{3} 2^{10} \pi^{2} \rho(\rho+2)^{2} \frac{E_{1}^{4}}{m k^{5}}\left(t^{2}(k)+\rho^{2}\right) \frac{\exp \left(\frac{4 \rho}{t(k)} \arctan (t k)\right)}{\exp \left(\frac{2 \pi \rho}{t(k)}\right)-1},
$$

where $t(k) \equiv \sqrt{k /\left|E_{1}\right|-1}, \rho \equiv 1 /\left(N_{c}^{2}-1\right)$ and $E_{1}$ is the binding energy of the $1 S$ state, this result was also obtained independently in [12]. A comparison between this cross section and the one found in [15] can be seen in fig. 1, the conclusion that can be drawn is that for $T \sim E_{1}$ or $T \ll E_{1}$ the obtained decay width is very sensitive to which cross section is used while for $T \gg E_{1}$ the ratio between the two cross sections is just a constant.

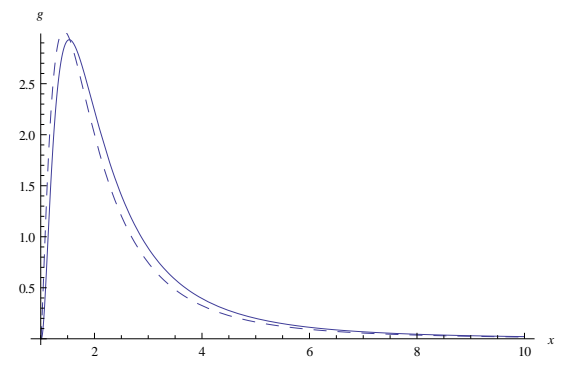

Figure 1: The function $g$, which is defined by $g\left(k /\left|E_{1}\right|\right)=\frac{3 \sigma_{1 S}(k)}{32 \pi C_{F} \alpha_{s} a_{0}^{2}}$ with $a_{0}$ the Bohr radius, is plotted. The dashed curve is the $N_{c} \rightarrow \infty$ curve normalized to the values of $a_{0}$ and $E_{1}$ of $N_{c}=3$ while the solid curve is the actual $N_{c}=3$ result.

The results of this subsection can be also found in a more extended way in [18].

\subsection{Quasi-free dissociation}

Quasi-free dissociation is the process $p+H Q \rightarrow p+Q+\bar{Q}$, the most commonly used cross section for this process were derived in [19]. Although this process is in principle suppressed by $\alpha_{s}$ compared to gluo-dissociation the phase-space for this process can be much larger if the temperature is high.

The cross section computed in [19] is actually the elastic scattering of heavy quarks $p+Q \rightarrow$ $p+Q$. On the phenomenological applications to heavy ions collisions [10] this cross section has been adapted to quasi-free dissociation of heavy quarkonium, $p+H Q \rightarrow p+Q+\bar{Q}$, by adding a quark/antiquark to $p+Q / \bar{Q} \rightarrow p+Q / \bar{Q}$ and modifying the masses to account for the binding energy. This approximation is equivalent to ignore the interference term shown in fig. 2 . In the EFT computation this interference term, which is of the same order of magnitude as the other ones, is also taken into account.

Apart from the cross section itself it is important to know how this is related to the decay width. According to the cutting rules [17], apart from the heavy quarks we have an in-coming and 


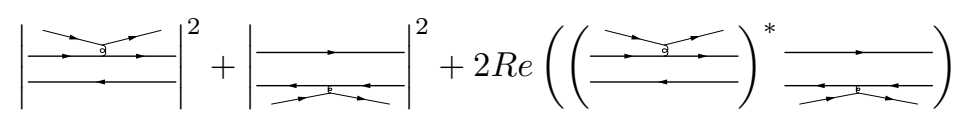

Figure 2: The imaginary part of the potential found in [5] seen as products of scattering matrix elements

an out-going parton, so we would expect a structure of the type

$$
\Gamma=\int \frac{d^{3} k}{(2 \pi)^{3}} n(k)(1 \pm n(k)) \sigma(k)
$$

which is different from the one used phenomenologically $[10,13,14]$. If an analysis of the previous EFT results is performed we found exactly the same result as the one obtained by cutting rules arguments.

Now on we show the results for the EFT cross section at different temperature regimes, for the time being we just discuss the process in which the parton is a fermion, the boson case is both quantitatively and qualitatively very similar. The highest temperature considered here is $T \gg 1 / r \sim$ $m_{D}$, in this regime the cross section is directly related to the imaginary part of the potential (that was first found in [5]), note however that the relation we have found between the decay width and the cross section assumes implicitly that the imaginary part is a perturbation, otherwise one should take into account the imaginary part of the potential as part of the unperturbed Hamiltonian in the same way as the Coulomb part is.

$$
\sigma_{1 S}^{q f}(k)=16 \pi C_{F} \alpha_{s}^{2} N_{F} a_{0}^{2}\left(1-\frac{4 m_{D}^{4} a_{0}^{4}-16+8 m_{D}^{2} a_{0}^{2} \log \left(\frac{4}{m_{D}^{2} a_{0}^{2}}\right)}{\left(m_{D}^{2} a_{0}^{2}-4\right)^{3}}\right),
$$

so one can see that when the momentum of the incoming parton is bigger that $1 / r$ the value of the cross section arrives to a plateau (it does not depend on $k$ ).

For the temperature regime $T \sim 1 / r \gg m_{D}$ we get

$$
\sigma_{1 S}^{q f}(k)=8 \pi C_{F} \alpha_{S}^{2} N_{F} a_{0}^{2}\left(-\frac{3}{2}+2 \log \left(\frac{2}{m_{D} a_{0}}\right)+\log \left(\frac{k^{2} a_{0}^{2}}{1+k^{2} a_{0}^{2}}\right)-\frac{1}{k^{2} a_{0}^{2}} \log \left(1+k^{2} a_{0}^{2}\right)\right),
$$

finally for $1 / r \gg T \gg m_{D} \gg E$ our result is

$$
\sigma_{1 S}^{q f}(k)=16 \pi C_{F} \alpha_{s}^{2} N_{F} a_{0}^{2}\left(\log \left(\frac{2 k}{m_{D}}\right)-1\right),
$$

this last result uses the color dipole approximation.

More details about the EFT approach to quasi-free dissociation will be found in [20].

\section{Quarkonium dissociation in a moving thermal bath}

Heavy quarkonium is believed to be produced in the early stages of the collision before the hot medium is formed, when heavy quarkonium interacts with the medium it can be affected in two ways, it can be drifted or it can be dissociated. In the last section we discussed dissociation when 

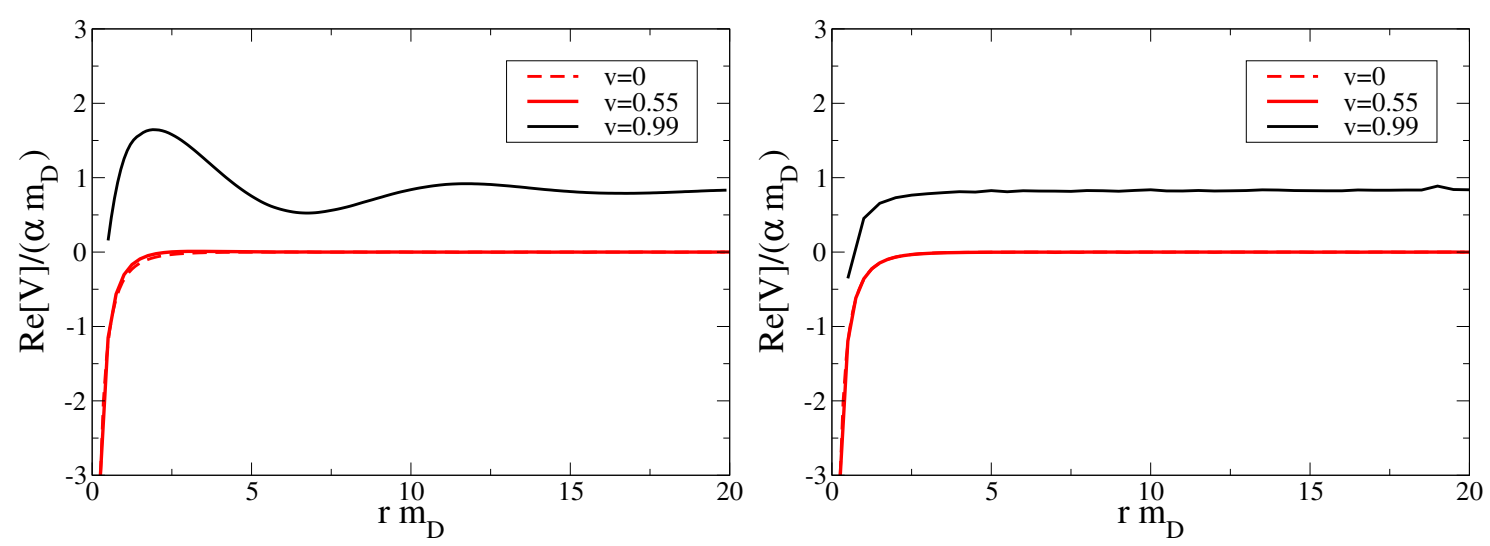

Figure 3: Real part of the potential at different velocities in the bound state rest frame. On the left panel we show the dependence in the direction parallel to the movement of the thermal bath while on the right we show the dependence on the perpendicular direction

the bound states is comoving with the thermal medium, now we are going to study what happens when they are not comoving. Although it has been seen that drift is important for open heavy flavor the situation may be different for heavy quarkonium. On one hand this is shown by the perturbative computation, on the other hand heavy quarkonium is a colorless particle whose size is very small while open heavy flavour states are not so small.

As before we use the techniques of EFTs to study this problem. This is done in an easier way by using the reference frame in which the bound state is at rest and the thermal bath is moving with a velocity $\mathbf{v}$. In this frame the Bose-Einstein (Fermi-Dirac) take the following form for massless particles

$$
f\left(\beta^{\mu} k_{\mu}\right)=\frac{1}{e^{\left|\beta^{\mu} k_{\mu}\right|} \pm 1},
$$

where $\beta^{\mu}=\frac{\gamma}{T}(1, \mathbf{v})$. The effect of this distribution can be intuitively understood by Doppler effect, one can define an effective temperature that depend on the direction of the particle. Approaching particles are seen with a bigger effective temperature while particles going away are seen with a smaller one

$$
T_{\mathrm{eff}}(\theta, v)=\frac{T \sqrt{1-v^{2}}}{1-v \cos \theta}
$$

We focus on the temperature regime $T \gg 1 / r \sim m_{D}$ which is the one that is relevant for dissociation (more details and other temperature regimes are analized in [21]). This was first study in [22], however only the real part of the potential was taken into account. In the comoving case dissociation is due to the imaginary part of the potential, so it is more interesting to consider what happens to this part, however we review the modifications to the real part for completeness. In fig. 3 the real part of the potential is shown for different velocities of the thermal bath in the direction parallel to the movement of the thermal medium and on the perpendicular one. It can be seen that at short distances the potential is more steep as the velocity increases and this means that the effect of screening is more important. Another important observation is that the dependence is anisotropic. On the parallel direction an oscillatory structure can be seen that is absent in the perpendicular case. 

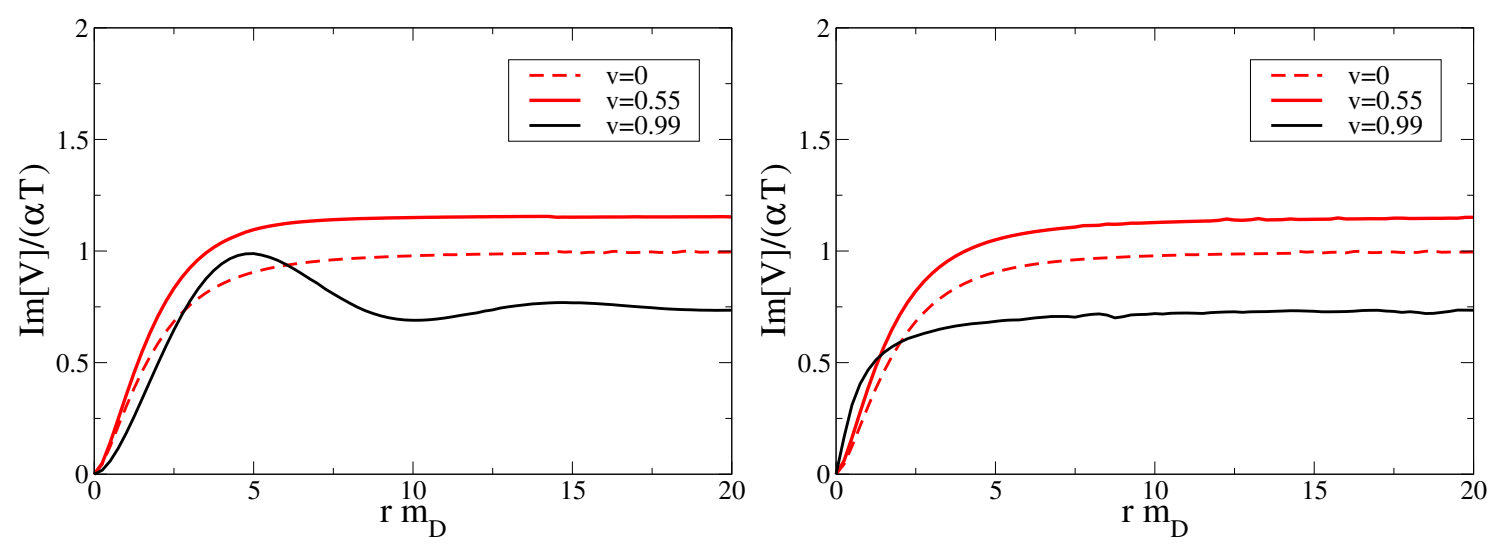

Figure 4: Imaginary part of the potential at different velocities in the bound state rest frame. On the left panel we show the dependence in the direction parallel to the movement of the thermal bath while on the right we show the dependence on the perpendicular direction

Next we discuss the imaginary part of the potential. The results that we found at different velocities can be seen in fig. 4. We can see that for moderate velocities the imaginary part of the potential always increases (this behavior is found up to $v=0.9$ ) but for very large velocities the situation is not so clear because the answer depends on the distance as well as the direction. The conclusion is that at moderate velocities dissociation will increase with the momentum. At very large velocities it seems that the imaginary part of the potential will not be so important and screening will be relevant to produce dissociation. The effects of this potential will be discussed in a more quantitative way in a future publication [23].

\section{Conclusions}

In these proceedings we have discussed some recent developments in the application of EFTs to the study of heavy quarkonium in the quark-gluon plasma. On section 2 we studied the relation between the results previously found in EFTs for the decay width and the results found by directly using $T=0$ cross sections in a thermal medium. We found that the EFTs results can also be written in the cross section language and allow us to go beyond some approximations (as for example the large $N_{c}$ approximation) that were used in the previous computations of these cross sections. Moreover EFT power counting makes it easier to know which approximations can be done at a given temperature regime. On section 3 we discussed how dissociation is modified when the bound state is moving with a given momentum. We found that dissociation increases with velocity up to $v \sim 0.9$. At very large velocities we see that screening effects will become more important.

\section{Acknowledgements}

I thank Nora Brambilla, Floriana Gianuzzi, Jacopo Ghiglieri, Massimo Mannarelli, Joan Soto and Antonio Vairo for collaboration on the work presented here. We acknowledge finantial support from the DFG project BR4058/1-1 "Effective field theories for strong interactions with heavy quarks". 


\section{References}

[1] T. Matsui and H. Satz, Phys. Lett. B 178 (1986) 416.

[2] N. Brambilla, S. Eidelman, B. K. Heltsley, R. Vogt, G. T. Bodwin, E. Eichten, A. D. Frawley and A. B. Meyer et al., Eur. Phys. J. C 71 (2011) 1534 [arXiv:1010.5827 [hep-ph]].

[3] M. A. Escobedo and J. Soto, Phys. Rev. A 78 (2008) 032520 [arXiv:0804.0691 [hep-ph]].

[4] N. Brambilla, J. Ghiglieri, A. Vairo and P. Petreczky, Phys. Rev. D 78 (2008) 014017 [arXiv:0804.0993 [hep-ph]].

[5] M. Laine, O. Philipsen, P. Romatschke and M. Tassler, JHEP 0703 (2007) 054 [hep-ph/0611300].

[6] N. Brambilla, M. A. Escobedo, J. Ghiglieri, J. Soto and A. Vairo, JHEP 1009 (2010) 038 [arXiv:1007.4156 [hep-ph]].

[7] G. Aarts, C. Allton, S. Kim, M. P. Lombardo, M. B. Oktay, S. M. Ryan, D. K. Sinclair and J. I. Skullerud, JHEP 1111 (2011) 103 [arXiv:1109.4496 [hep-lat]].

[8] J. Ghiglieri, on these proceedings.

[9] D. Kharzeev and H. Satz, Phys. Lett. B 334 (1994) 155 [hep-ph/9405414].

[10] L. Grandchamp and R. Rapp, Phys. Lett. B 523 (2001) 60 [hep-ph/0103124].

[11] X. Zhao and R. Rapp, Phys. Rev. C 82 (2010) 064905 [arXiv:1008.5328 [hep-ph]].

[12] F. Brezinski and G. Wolschin, Phys. Lett. B 707 (2012) 534 [arXiv:1109.0211 [hep-ph]].

[13] F. Nendzig and G. Wolschin, arXiv:1210.8366 [hep-ph].

[14] T. Song, K. C. Han and C. M. Ko, Phys. Rev. C 85 (2012) 014902 [arXiv:1109.6691 [nucl-th]].

[15] G. Bhanot and M. E. Peskin, Nucl. Phys. B 156 (1979) 391.

[16] N. Brambilla, A. Pineda, J. Soto and A. Vairo, Rev. Mod. Phys. 77 (2005) 1423 [hep-ph/0410047].

[17] R. L. Kobes and G. W. Semenoff, Nucl. Phys. B 272 (1986) 329.

[18] N. Brambilla, M. A. Escobedo, J. Ghiglieri and A. Vairo, JHEP 1112 (2011) 116 [arXiv:1109.5826 [hep-ph]].

[19] B. L. Combridge, Nucl. Phys. B 151 (1979) 429.

[20] N. Brambilla, M. A. Escobedo, J. Ghiglieri and A. Vairo, preprint number TUM-EFT 27/11, in preparation.

[21] M. A. Escobedo, J. Soto and M. Mannarelli, Phys. Rev. D 84 (2011) 016008 [arXiv:1105.1249 [hep-ph]].

[22] M. C. Chu and T. Matsui, Phys. Rev. D 39 (1989) 1892.

[23] M. A. Escobedo, F. Gianuzzi, M. Mannarelli and J. Soto, preprint number TUM-EFT 35/12, in preparation. 